\title{
Nonsense Mutations of the Ornithine Decarboxylase Structural Gene of Neurospora crassa
}

\author{
ROWLAND H. DAVIS, * LORE V. HYNES, AND PAMELA EVERSOLE-CIRE \\ Department of Molecular Biology and Biochemistry, University of California at Irvine, Irvine, California 92717
}

\author{
Received 30 September 1986/Accepted 1 December 1986
}

\begin{abstract}
Ornithine decarboxylase (ODC) (EC 4.1.1.17) is an early enzyme of polyamine synthesis, and its activity rises quickly at the onset of growth and differentiation in most eucaryotes. Some have speculated that the enzyme protein may have a role in the synthesis of rRNA in addition to its role in catalyzing the decarboxylation of ornithine (G. D. Kuehn and V. J. Atmar, Fed. Proc. 41:3078-3083, 1982; D. H. Russell, Proc. Natl. Acad. Sci. USA 80:1318-1321, 1983). To test this possibility, we sought mutational evidence for the indispensability of the ODC protein for normal growth of Neurospora crassa. We found three new, ODC-deficient mutants that lacked ODC protein. Among these and by reversion analysis of an earlier set of mutants, we found that two ODC-deficient mutants carried nonsense mutations in the ODC structural gene, spe-1. Allele LV10 imparted a complete deficiency for enzyme activity $(<0.006 \%$ of normal) and had no detectable ODC antigen. Allele PE4 imparted a weak activity to cells $\left(0.1 \%\right.$ of derepressed $s p e^{+}$cultures) and encoded a lower-molecular-weight ODC subunit $\left(M_{\mathrm{r}}=43,000\right)$ in comparison to that of the wild-type strain $\left(M_{\mathrm{r}}=53,000\right)$. Strains carrying either mutation, like other spe-1 mutants, grew at a normal rate in exponential culture if the medium was supplemented with spermidine, the main end product of the polyamine pathway in $N$. crassa. Unless an antigenically silent, $\mathrm{N}$-terminal fragment with an indispensable role persists in the LV10-bearing mutant, we conclude that the ODC protein has no role in the vegetative growth of this organism other than the synthesis of polyamines. The data extend earlier evidence that spe-1 is the structural gene for ODC in $N$. crassa. The activity found in mutants bearing allele PE4 suggests that the amino acids nearest the carboxy terminus do not contribute to the active site of the enzyme.
\end{abstract}

Ornithine decarboxylase (ODC), an initial enzyme of polyamine synthesis, is the only route of putrescine synthesis in fungi and animals (23). Augmentation of ODC activity is associated with the onset of growth and differentiation in most organisms. The enzyme is rapidly inactivated as growth stops or when polyamines are added to cells with high activity. Some investigators have hypothesized that ODC may have a role in the growth process besides its known catalytic activity. Kuehn and Atmar (13) have presented evidence that an inactive, phosphorylated form of ODC participates in rRNA synthesis in the slime mold Physarum polycephalum. A similar idea was suggested by Russell (19) in studies of the early stages of embryogenesis in the amphibian Xenopus laevis.

If these ideas are correct, the ODC protein should be indispensable to normal growth and mutants lacking the protein should be abnormal or inviable even if polyamines are provided to them. Mutants lacking ODC enzyme activity are now known for a number of eucaryotic organisms $(5,10$, $16,18,20-22,27)$, but only in Neurospora crassa has a critical identification of the structural gene been provided by mutational analysis (10). In that case, however, no mutation was shown to lack both enzyme activity and the protein.

The present study was designed to isolate nonsense mutants of ODC which demonstrably lack both ODC activity and protein and to assess the ability of such mutants to grow. In addition, revertants of missense mutations were collected in hopes of finding forms of the enzyme altered in the inactivation process.

\footnotetext{
* Corresponding author.
}

\section{MATERIALS AND METHODS}

Strains, media, and stock maintenance. The strains of $N$. crassa used here, including the new spe-l mutants collected in this work, are shown in Table 1 . The inl and his mutations were grown with supplements of 50 and $100 \mu \mathrm{g}$ of their respective supplements, inositol and histidine. Spermidine trihydrochloride was used as a stock supplement for spe-1 (ODC deficient) mutants at $250 \mu \mathrm{g} / \mathrm{ml}$ of medium. Vogel medium N (26) was used for stock maintenance and growth experiments. Synthetic crossing medium or corn meal agar medium (Difco Laboratories) was used for crosses (6).

Mutagenesis and outcrossing. The basic genetic techniques used here have been described previously (6). New ODCless mutants of the LV series were isolated from UV-treated conidia of the aga inl double mutant, strain IC-40. The inositol-less death method (15), modified slightly from the protocol used previously (10), was used. The conidia used for selection were grown before mutagenesis in medium that leads to partial polyamine starvation (10). After UV mutagenesis, leaving $45 \%$ as survivors, selection was carried out at $35^{\circ} \mathrm{C}$ in medium that permits outgrowth of conidia in a very low level of inositol $(0.25 \mu \mathrm{g}$ per $\mathrm{ml})(10)$. This medium also contained $0.05 \mathrm{mM}$ methylglyoxal-bis-guanylhydrazone. Addition of this compound, an inhibitor of spermidine synthase, was found to impair growth only slightly at the concentration used, but it led to a more rapid expression of the polyamine-dependent phenotype of newly induced mutants. Surviving cells were plated after 48 -h selection (and periodic filtrations through cheesecloth to remove clumped mycelium) on medium containing $250 \mu \mathrm{g}$ of spermidine trihydrochloride and $50 \mu \mathrm{g}$ of inositol per ml. They were later spot tested (6) on minimal medium or on media con- 
TABLE 1. Strains used in this study

\begin{tabular}{lll}
\hline \multicolumn{1}{c}{ Strain } & \multicolumn{1}{c}{$\begin{array}{c}\text { Genotype }{ }^{a} \text { (with spe-l } \\
\text { allele) }\end{array}$} & Source \\
\hline IC-3 & $\begin{array}{l}\text { aga A } \\
\text { aga, inl a }\end{array}$ & R. Davis \\
IC-40 & his-1 a & R. Davis \\
IC-39 & his-1 A & FGSC \\
IC-1106-18 & his-1 a & R. Davis \\
FGSC-680 & his-1 eas A & FGSC \\
IC-48 & his-1 ssu-1 A & D. Perkins \\
IC-2249-7 & spe-1 aga (462JM) A & R. Davis \\
IC-1241-6 & spe-1 aga $(521 \mathrm{KW})$ A & R. Davis \\
IC-41 & spe-1 aga (521KW) a & R. Davis \\
IC-1242-52 & spe-1 aga (TP138) A & R. Davis \\
IC-9 & spe-1 (TP138) a & R. Davis \\
IC-752-8 & spe-1 aga (PE4) A & R. Davis \\
IC-1474-13 & spe-1 aga (PE4) a & R. Davis \\
IC-1474-104 & spe-1 aga (PE7) A & R. Davis \\
IC-44 & spe-1 aga (PE7) a & R. Davis \\
IC-1475-3 & spe-1 aga (PE69) A & R. Davis \\
IC-1476-145 & spe-1 aga (PE85) A & R. Davis \\
IC-1477-184 & spe-1 aga (PE85) a & R. Davis \\
IC-1477-122 & spe-1 aga (LV10) a & R. Davis \\
IC-1894-53 & spe-1 aga, inl (LV17) a & This paper \\
IC-1895-4 & spe-1 (LV71) a & This paper \\
IC-1896-22 & spe-1 (LV86) a & This paper \\
IC-1897-24 & spe-1 inl (LV155) a & This paper \\
IC-1900-14 & spe-1 inl (LV170) A & This paper \\
IC-1901-14 & spe-1 inl (LV206) a & This paper \\
IC-1903-28 & This paper \\
\hline
\end{tabular}

a Standard alleles used: aga, UM906; inl, 89601; his-1, Y155M302; ssu-1, Y319-44; eas, UCLA191.

${ }^{b}$ FGSC, Fungal Genetics Stock Center, Department of Microbiology, University of Kansas Medical Center, Kansas City.

taining putrescine or spermidine at 25 and $35^{\circ} \mathrm{C}$ to identify unambiguous mutants.

New, polyamine-dependent mutants were tested for complementation with a strain carrying the classical spe-I mutation, 462JM (16). Noncomplementers were outcrossed to a wild-type strain to test for the linkage, expected of spe-1 alleles, to the inl mutation. The outcross allowed isolation of the new mutations with various combinations of the inl and aga mutations. A problem encountered regularly with polyamine-dependent mutants is the very poor germination of ascospores (18), a problem mitigated to some extent by the use of $500 \mu \mathrm{g}$ of spermidine trihydrochloride per ml of plating medium.

Revertants were isolated from conidia of spe-1 mutants carrying the aga mutation (see below) by treatment with UV light and were then plated on medium lacking polyamine supplementation. Colonies were transferred to individual tubes, purified by one or two rounds of single-conidial isolation, and outcrossed to strains of the opposite mating type that carried the spe-1 mutation from which given revertant alleles were derived. $\mathrm{Spe}^{+} \mathrm{Aga}^{-}$progeny of the outcrosses were selected; they were homocaryotic and suitable for further analysis.

Further genetic analysis of revertants. Reversion could be intragenic or it could be due to the occurrence of an easily recombining, extragenic suppressor (e.g., a nonsense suppressor). The following strategy was used to distinguish these cases and to deal at the same time with the very poor germination of polyamine-dependent strains.

The his-1 mutation lies within 1 map unit of the spe-1 locus. In mating each outcrossed revertant to the his-1spe $-1^{+}$strain and plating the ascospores on medium containing spermidine but no histidine, it could be determined whether the original spe-1 mutation was present in the revertant. The absence of polyamine-dependent mutants in such crosses signifies an intragenic reversion or a suppressor mutation in a gene closely linked to the spe-1 mutation. This test was compromised by the poor germination of spe-1 strains, but selection against his- $1^{-}$and thus against spe-1 ${ }^{+}$ made the test adequate for our purposes.

The appearance of spermidine-dependent colonies in this cross signifies that reversion has taken place by the occurrence of a suppressor mutation elsewhere in the genome. Where this occurred (see Results), strains carrying the suppressor mutation in a spe- $1^{+}$background were sought in another plating among the $\mathrm{His}^{-}$segregants, almost all of which (at least $99.5 \%$ ) should contain the wild-type spe-1 allele. Strains carrying the suppressor were identified by test-crossing a number of these $\mathrm{His}^{-}$segregants with a strain (genotype spe-1 $1^{-}$his- $\mathrm{I}^{+}$) carrying the original, presumptively suppressible spe-1 allele and by plating on minimal medium to detect $\mathrm{His}^{+}$progeny that were also $\mathrm{Spe}^{+}$. Only when the suppressed mutant could be reconstructed in the latter cross was the evidence for a suppressor mutation judged to be conclusive.

Extremely poor germination in the crosses used to identify suppressor-carrying $\mathrm{His}^{-}$strains led us to use the total number of spores plated, rather than the number of germinated spores, to calculate the percentage of $\mathrm{Spe}^{+}$progeny. This was justified by the ability of $\mathrm{Spe}^{+}$spores to synthesize polyamines, germinate reasonably well, and thus distinguish themselves from $\mathrm{Spe}^{-}$progeny, germinated or ungerminated. $\mathrm{Spe}^{+}$colonies arising by recombination between spe-1 and his-1 were expected at a level of $0.5 \%$ in all crosses. Such strains involving a suppressor-bearing parent, however, would generate prototrophs at a higher level (up to $50 \%$ of progeny bearing the spe-1 mutation). For pedigrees suggesting the occurrence of a suppressor mutation, further test-crossing of a progeny sample was done to confirm the genotypes.

ODC and protein assays and polyamine determination. The activity of ODC was determined in extracts ground with sand and prepared as in previous work (10). Certain later work reported here used extracts of acetone powders, which were easy to prepare, store, and extract (6) and had ODCspecific activities indistinguishable from sand-ground extracts. ODC assays were carried out as described earlier (18) with $2 \mathrm{mM}$ dithiothreitol substituted for 2-mercaptoethanol in most cases. For determination of very low activities of mutant strains, the specific radioactivity of the substrate was raised by a factor of 5 or 10 . We defined $1 \mathrm{U}$ of activity as 1 nmol of $\mathrm{CO}_{2}$ evolved per $\mathrm{h}$ at $37^{\circ} \mathrm{C}$. Proteins were determined by the method of Bradford (2). Polyamines extracted from cells were determined by high-performance liquid chromatography methods as described previously (7).

Immunological methods. Western immunoblotting was done by standard methods (3). The antibody used was raised to ODC purified to the penultimate stage of purification (lacking only the high-performance liquid chromatography step) (7, 10; J. J. DiGangi, M. Seyfzadeh, and R. H. Davis, manuscript submitted). The preparation was denatured and subjected to sodium dodecyl sulfate-polyacrylamide gel electrophoresis (14), in either uniform $10 \%$ or 7.5 to $15 \%$ gradient gels. The main band of ODC was cut out, eluted from the polyacrylamide, and used as an antigen. The antiserum made against the ODC preparation reacted to ODC bands and one non-ODC band in immunoblots of gels of crude, wild-type extracts. The antiserum was rendered specific without a detectable loss of titer by cross-absorbing the crude serum 
with immobilized extracts of a strain carrying the nonsense mutation, LV10 (see Results). The extract was made from a culture of this strain grown in limiting putrescine $(0.2 \mathrm{mM})$ and containing $1 \mathrm{mM}$ arginine. (This duplicates the conditions of polyamine starvation and arginine abundance characteristic of the culture from which ODC was purified [7].) The crude extract, from sand-ground mycelium, was coupled to CNBr-activated Sepharose 4B (28). Before an antiserum was used, it was exposed to a substantial excess of the immobilized, ODC-less extract for $40 \mathrm{~h}$ in the cold, a treatment that did not reduce the titer of the antibody against pure ODC or the ODC of crude extracts.

Extracts for immunoblotting analysis were made from strains grown in media with limiting putrescine $(0.2 \mathrm{mM})$. The controls used were (i) pure ODC; (ii) extracts made as above of strain IC-1894-53, which carries the nonsense spe-1 allele, LV10; or (iii) extracts of the arginase-less strain, IC-3, grown in $1 \mathrm{mM}$ arginine to derepress ODC. In these conditions, strain IC-3 is unable to synthesize ornithine by the catabolic (mutant) or biosynthetic (feedback inhibited) route. The resulting deprivation of polyamines leads to high levels of ODC activity and ODC protein $(7,17)$.

Materials. Most chemicals were from Sigma Chemical Co. Sepharose 4B was from Pharmacia, and the Bradford protein assay reagents were from Bio-Rad Laboratories. ${ }^{125}$ I-protein A for use in immunoblotting was a gift of Daniel Knauer.

\section{RESULTS}

Isolation of new mutants. Seven new mutants with severe or complete ODC deficiencies were isolated. None complemented with a bona fide spe-1 strain. The ODC-less determinant was linked in all cases to the inl mutation in outcrosses. The strains grew well on putrescine and spermidine but poorly or not at all on minimal medium. These data certify the seven mutants as spe-1 mutants. Their phenotypes are summarized in Table 2 .

A Western immunoblot of six new mutant extracts, together with previously isolated strains $(10,16,18)$ carrying the alleles PE4, PE7, PE69, PE85, 462JM, and 521KW, is shown in Fig. 1, and the results are also indicated in Table 2. Seven mutants display a strong $M_{\mathrm{r}}$ band of 52,000 to 54,000 , similar to the positive (polyamine-starved) control and to one of the bands of pure ODC. (Two other mutants carrying

TABLE 2. Growth, enzyme, and immunoblot information on new spe- 1 alleles ${ }^{a}$

\begin{tabular}{lccccc}
\hline \multirow{2}{*}{$\begin{array}{c}\text { spe-l } \\
\text { allele }\end{array}$} & \multicolumn{2}{c}{$\begin{array}{c}\text { Growth }^{b}(\mathrm{mg} \text { [dry wt]/10 ml) } \\
\text { on: }\end{array}$} & $\begin{array}{c}\text { ODC sp act (U/mg } \\
\text { of protein })^{c}\end{array}$ & $\begin{array}{c}\text { Protein in } \\
\text { immuno- } \\
\text { blot }\left(M_{\mathrm{r}}\right)^{d}\end{array}$ \\
\cline { 2 - 4 } & MIN & MIN + PUT & MIN + SPD & & \\
\hline spe-1 & 29 & 25 & 29 & 1,550 & 53,000 \\
LV10 & 0 & 34 & 35 & $<0.1$ & none \\
LV17 & 1 & 33 & 33 & 0.1 & 53,000 \\
LV71 & 1 & 31 & 35 & $<0.1$ & none \\
LV86 & 8 & 35 & 38 & 0.4 & 53,000 \\
LV155 & 1 & 34 & 34 & $<0.1$ & 53,000 \\
LV170 & 0 & 26 & 28 & $<0.1$ & none \\
LV206 & 2 & 39 & 40 & 0.1 & 53,000 \\
\hline
\end{tabular}

${ }^{a}$ Growth and enzyme data on other spe-l alleles (PE4, PE7, PE69, PE85, TP138, 469JM, and $521 \mathrm{KW}$ ) can be found in references 10 and 18 and Fig. 1.

${ }^{b}$ Growth was measured in 10-ml stationary cultures grown for $48 \mathrm{~h}$ at $25^{\circ} \mathrm{C}$ in minimal medium (MIN) or MIN supplemented with $1 \mathrm{mM}$ putrescine (PUT) or $1 \mathrm{mM}$ spermidine (SPD).

${ }^{c}$ Enzyme activities were measured in sand-ground extracts of exponentially growing cultures maintained at $25^{\circ} \mathrm{C}$ in the presence of limiting $(0.2 \mathrm{mM})$ putrescine.

${ }^{d}$ Immunoblot data are from Fig. 1, except earlier data for LV17.

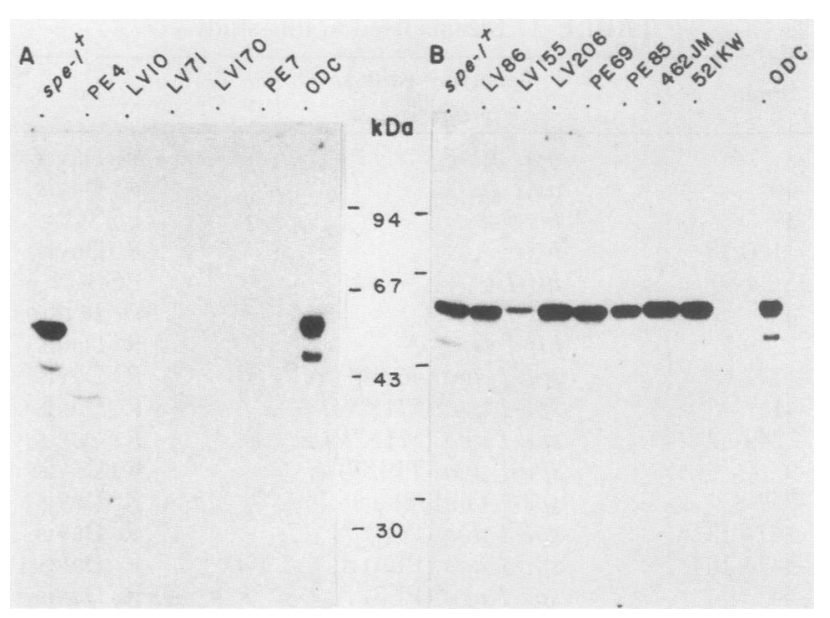

FIG. 1. Immunoblots of extracts of mutants bearing spe-1 mutations. The allele numbers are given at the top of the corresponding lane of the gel. Controls were pure ODC and an extract of derepressed strain IC-3 $\left(\right.$ spe $\left.-I^{+}\right)$. Preimmu..e serum recognized no polypeptide of extracts of the latter strain. Indicated are mutants without (A) and with (B) a polypeptide of $53,000 M_{r}$.

alleles LV17 and TP138 displayed normal ODC bands but are not shown.) The mutants with ODC polypeptide also displayed a faint band of 47,000 $M_{\mathrm{r}}$ (not visible in Fig. 1) commonly seen in the control and in pure ODC preparations, the latter band arising as a proteolytic artifact during purification (DiGangi et al., submitted).

Of the 12 mutants, 5 lacked protein of the normal molecular weight $(53,000)$ (Table 2). Three of these mutants, carrying alleles LV10, LV71, and LV170, are from the new series, and two, carrying the alleles PE4 and PE7, are from the previous series (10). PE4- and PE7-carrying mutants were not expected to lack ODC protein, because they had residual enzyme activity and grew slowly in minimal medium (10). However, the mutant carrying the PE4 allele displayed a lower-molecular-weight band on the immunoblot $\left(M_{\mathrm{r}}\right.$, ca. $43,000)$. The high specificity of the antiserum suggests strongly that it is a fragment of the ODC polypeptide, and in fact, as shown below, PE4 is a nonsense mutation. The PE7 mutation, in previous careful tests of the strain carrying it, does not impart a complete ODC deficiency (10). Thus it is likely that the PE7 allele encodes a form of ODC that is too little or labile to visualize on gels.

Reversion of spe-1 alleles. Some of the previously available alleles (462JM, 521KW, TP138, PE4, PE7, PE69, and PE85) were tested for reversion. Several revertants from each mutant were collected and characterized (Table 3). Extracts of all revertants displayed activity on minimal medium, some having about as much activity as a wild-type (or agacarrying) strain on the same medium. Growth of the revertants, all carrying the aga mutation, in arginine-containing medium allowed a test of the maximal extent of ODC derepression. In this medium, polyamine starvation ensues owing to ornithine depletion. A large variation in ODC activity was seen among the revertants, indicating that the enzyme-forming potential regained by reversion was in many cases impaired and was probably already derepressed in cells grown in minimal medium (Table 3 ). In such revertants, the capacity for further derepression was thus limited. This view was supported by the observation that spermidine, which reduces the activity of normal mycelia by about $40 \%$, reduced ODC activity of the partial revertants to undetect- 
TABLE 3. Phenotypes of selected revertants showing ODC activity on different media

\begin{tabular}{|c|c|c|c|c|}
\hline \multirow{2}{*}{$\begin{array}{l}\text { Parent allele and } \\
\text { revertant strain }\end{array}$} & \multicolumn{3}{|c|}{ ODC activity (U/mg of protein) ${ }^{b}$} & \multirow{2}{*}{$\begin{array}{l}\text { Spermidine content } \\
\text { (nmol/mg of } \\
\text { protein) }\end{array}$} \\
\hline & MIN & MIN + ARG & MIN + SPD & \\
\hline \multicolumn{5}{|l|}{ TP138 } \\
\hline IR101-R2 & 29 & - & - & - \\
\hline IR102-Rla & 35 & 464 & - & - \\
\hline IR103-R1 & 36 & - & - & - \\
\hline IR105-R4 & 37 & - & - & - \\
\hline \multicolumn{5}{|l|}{$521 \mathrm{KW}$} \\
\hline IR111-R1 & 8 & 13 & 4 & 2.7 \\
\hline IR113-R2 & $<1$ & 1 & $<1$ & 1.6 \\
\hline IR114-R1 & 3 & 59 & $<1$ & 7.8 \\
\hline IR115-R2 & 21 & 51 & 6 & 8.5 \\
\hline IR117-R1 & 32 & 109 & 6 & 8.5 \\
\hline IR118-R2 & 9 & 33 & 4 & 9.2 \\
\hline \multicolumn{5}{|l|}{ PE4 } \\
\hline IR121-R2 & 4 & 67 & 2 & 12.8 \\
\hline IR123-R2 & 5 & 6 & 0 & 8.9 \\
\hline IR127-R1 & 5 & 8 & $<1$ & 9.5 \\
\hline \multicolumn{5}{|l|}{ PE7 } \\
\hline IR131-R1 & 39 & 487 & 7 & 15.1 \\
\hline IR132-R2 & 15 & 166 & - & 11.6 \\
\hline IR133-R1 & 24 & 173 & 5 & 11.8 \\
\hline IR135-R7 & 13 & 177 & 3 & 18.1 \\
\hline \multicolumn{5}{|l|}{ PE85 } \\
\hline IR141-R1 & 27 & 1,549 & 7 & 12.4 \\
\hline \multicolumn{5}{|l|}{ Control $\left(\right.$ spe- $\left.1^{+}\right)$} \\
\hline IC-3 & 21 & 1,600 & 16 & 14.0 \\
\hline
\end{tabular}

a Specific activities of strains carrying parent alleles, when grown in conditions of polyamine starvation were for each allele as follows: TP138, <0.1; 521KW, $<0.1$; PE4, 2.3; PE7, 1.8; and PE85, 3.9. The comparable value range for an spe-1+ culture was 1,200 to 1,800 .

$b$ Abbreviations: MIN, minimal medium; ARG, $1 \mathrm{mM}$ arginine hydrochloride; SPD, $1 \mathrm{mM}$ spermidine trihydrochloride.

c For strains grown in minimal medium.

able levels. In fact, most strains with impaired ODC activity in arginine-supplemented medium were spermidine deficient when grown in minimal medium (Table 3). The impaired ODC activity of many revertants suggested that second-site mutational events within the spe-1 gene underlay their phenotypes.

The large majority of revertants, when analyzed for the linkage of the reversion event to the original mutation, appeared to be due to intragenic mutational events. In one intragenic revertant tested, the partially restored activity was regulated normally (Fig. 2). Attention was directed then to two revertants of the PE4-bearing strain that preliminary genetic analysis suggested might carry extragenic suppressor mutations.

Two revertant strains (with original designations of IR-123 and IR-127; Table 3) of the PE4-bearing strain yielded Spe ${ }^{-}$ progeny when crossed with a spe- $1^{+}$his $-1^{-}$strain. The His ${ }^{+}$ class segregated $1 \mathrm{Spe}^{-}: 1 \mathrm{Spe}^{+}$progeny. This demonstrated that unlinked mutations were responsible for suppressing the auxotrophy of the PE4 allele. Strains carrying the suppressor mutations (designated $s u^{123}$ and $s u^{127}$ ) were then sought among the $\mathrm{His}^{-}$siblings (i.e., spe- $1^{+}$his- $1^{-}$progeny) of the foregoing cross.

One half of these strains, when mated with a PE4-bearing strain $\left(\right.$ spe $-1^{-}$his- $\left.1^{+}\right)$, yielded progeny of which over $2 \%$ were $\mathrm{Spe}^{+} \mathrm{His}^{+}$(Table 4). Most of these progeny were presumably suppressed mutants (i.e., spe- $I^{-}$his $-I^{+} s u^{-}$; see Materials and Methods). In these crosses, less than the expected $50 \%$ frequency of $\mathrm{Spe}^{+} \mathrm{His}^{+}$prototrophs was seen; evidently germination was still impaired in the suppressed mutants. The other half of the strains yielded progenies with less than $1 \%$ of prototrophs, all of which are presumed to have arisen by recombination between spe-1 and his-1 (Table 4). The frequency of prototrophs expected from this mechanism was $0.5 \%$ (see Materials and Methods).

To test for the nonsense character of candidate spe-1 alleles, strains carrying the mutations were mated to a strain containing the his-1 mutation and the authentic nonsense suppressor mutation, ssu-1 $(4,8,9)$. The crosses had the same form as the ones used to verify the existence of the new suppressor mutations, $s u^{123}$ and $s u^{127}$. Again, the crosses yielded only a few germinating spores. Clear evidence of suppression was obtained for alleles PE4 and LV10, the latter causing a complete ODC protein deficiency (Fig. 1). The test was negative for allele LV71, which also imparted an ODC protein deficiency (Fig. 1). The data demonstrate that PE4 and LV10 are nonsense mutations and are consistent with the lower molecular weight of the PE4 product and the absense of detectable product in LV10-bearing strains. By extension, the mutations $s u^{123}$ and $s u^{127}$ are nonsense suppressor mutations. Crosses to test the effect of these suppressor mutations on the other spe-1 alleles were largely sterile.

Immunoblot analysis of suppressed mutants was performed. Extracts of suppressed mutants grown in minimal medium and thus effectively limited for polyamines yielded a 
smear of antigenic material in the molecular weight range of 47,000 to 90,000 on the nitrocellulose blots (Fig. 3). By varying conditions, we saw this effect only in materials with a suppressor mutation $\left(s s u-1, s u^{123}\right.$, and $s u^{127}$ imparted the effect equally) and demonstrable ODC activity. In some cases, the activity was that restored by the suppressor mutation: unsuppressed LV10-bearing strains did not yield the effect, whereas cells of the LV10 ssu- 1 genotype did (Fig. $3)$. Moreover, cells carrying only the $s s u-1$ mutation displayed the effect even if they were grown in minimal medium, but as in the case of in vitro ODC activity, the signal on the immunoblot was weak (data not shown). This eliminated spermidine starvation in suppressor-bearing strains as a sufficient condition for the effect. The origin of the material of low and heterogeneous mobility is not known; it may have been owing to precipitation of ODC monomers during electrophoresis, possibly caused by readthrough $\mathrm{C}$-terminal amino acids beyond the normal stop codon. Such an effect was not seen for a suppressed-mutant, mitochondrial enzyme (encoded in the nucleus) in our previous work with the particular $s s u-l$ mutation used (9). The effect was not seen among nonspecific antigens when a much less specific antiserum was used to probe blots. Whatever the implication of the immunoblot analysis, the return of enzyme activity and the ability of suppressed mutants to grow are evidence that the suppressor genes counter the chain-terminating action of the PE4 and LV10 nonsense mutations.

Growth rates of nonsense mutants. A comparison of growth rates was made in spermidine-supplemented medium of strains carrying the $s p e-1^{+}$allele or the nonsense mutations LV10 and PE4 (Fig. 4). The similar growth rates of the three strains shows that ODC protein is not required for vegetative
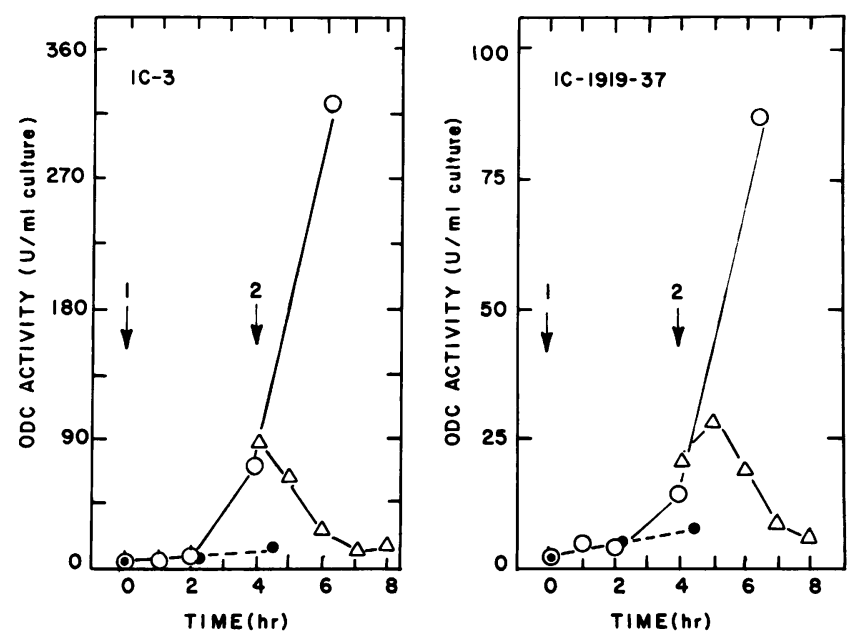

FIG. 2. Behavior of ODC activity of strain IC-3 $\left(\right.$ spe- $\left.1^{+}\right)$and of a presumed intragenic revertant, strain IC-1919-37. The latter was selected from the PE7-bearing strain, IC-44, as IR132-R2 (Table 3). Strains were grown from conidial inocula in minimal medium, and at $0 \mathrm{~h}$, after we reserved some of each culture for further growth in minimal medium, arginine was added to a final concentration of 1 $\mathrm{mM}$ (arrow 1). At $4 \mathrm{~h}$, ornithine was added to a sample of the arginine-treated culture to a final concentration of $5 \mathrm{mM}$ to restore synthesis of polyamines (arrow 2). The remainder of the culture continued to grow in the presence of arginine alone. The ordinates of enzyme units per milliliter of culture have been adjusted to emphasize the similarity of enzyme regulation in the two strains. Symbols: -, growth on minimal medium; $O$, growth after addition of arginine; $\Delta$, growth after addition of ornithine to arginine-treated culture.
TABLE 4. Identification of suppressor-bearing strains among progeny of a cross between suppressed mutant IR123-3 and his-1 mutant IC- $48^{a}$

\begin{tabular}{|c|c|c|c|c|}
\hline \multirow{2}{*}{$\begin{array}{c}\text { Suppressor } \\
\text { presence } \\
\text { and strain no. }\end{array}$} & \multirow{2}{*}{$\begin{array}{l}\text { Spores } \\
\text { plated }\end{array}$} & \multicolumn{2}{|c|}{ Colonies on ${ }^{c}$ : } & \multirow{2}{*}{$\begin{array}{c}\% \\
\text { Prototrophs }\end{array}$} \\
\hline & & MIN & $M I N+S P D$ & \\
\hline \multicolumn{5}{|l|}{ Suppressor $(+)$} \\
\hline $1785-11$ & 1,640 & 109 & 158 & 7 \\
\hline $1785-12$ & 1,320 & 68 & 102 & 5 \\
\hline $1817-13$ & 1,220 & 32 & 34 & 3 \\
\hline $1817-14$ & 2,100 & 39 & 77 & 2 \\
\hline $1817-16$ & 1,180 & 58 & 64 & 5 \\
\hline $1817-24$ & 2,560 & 261 & 247 & 10 \\
\hline $1817-31$ & 320 & 6 & 42 & 2 \\
\hline $1817-32$ & 1,360 & 53 & 43 & 4 \\
\hline $1817-36$ & 1,620 & 103 & 93 & 6 \\
\hline $1817-37$ & 1,500 & 169 & 167 & 11 \\
\hline \multicolumn{5}{|l|}{ Suppressor (-) } \\
\hline $1785-13$ & 590 & 3 & 9 & 0.5 \\
\hline $1785-19$ & 1,030 & 3 & 39 & 0.3 \\
\hline $1817-9$ & 150 & 1 & 3 & 0.7 \\
\hline $1817-12$ & 1,080 & 4 & 11 & 0.4 \\
\hline $1817-15$ & 130 & 0 & 11 & 0 \\
\hline $1817-23$ & 2,200 & 2 & 121 & 0.1 \\
\hline $1817-27$ & 1,180 & 5 & 45 & 0.4 \\
\hline $1817-29$ & 1,060 & 7 & 33 & 0.7 \\
\hline $1817-33$ & 1,400 & 6 & 40 & 0.4 \\
\hline $1817-34$ & 2,200 & 5 & 32 & 0.2 \\
\hline $1817-35$ & 980 & 5 & 60 & 0.5 \\
\hline
\end{tabular}

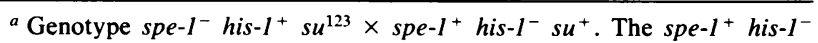
progeny were selected (as $\mathrm{Spe}^{+} \mathrm{His}^{-}$). These strains, crossed to a strain (spe- $1^{-}$his $-I^{+}$) carrying the suppressible allele PE4, were predicted to segregate in a ratio of 1 suppressor-bearing to 1 suppressor-free strain. Spores were plated on minimal medium to determine the number of $\mathrm{Spe}^{+} \mathrm{His}^{+}$ prototrophs arising through suppression of the spermidine requirement imposed by allele PE4 or by recombination between spe-l and his-l (expected at $0.5 \%$ in all crosses).

${ }^{b}$ Indicates putative suppressor-bearing $(+)$ or -less $(-)$ strains.

c Abbreviations: MIN, minimal medium; SPD, $1 \mathrm{mM}$ spermidine trihydrochloride.

growth if spermidine is provided. This confirms data from stationary-growth tests reported in Table 2.

\section{DISCUSSION}

We have shown with new mutations and reversion analysis that the structural gene for ODC can be readily mutated to forms that lead to the loss of detectable ODC protein from cells. This conclusion is based on the specific antiserum developed against purified ODC. Inasmuch as the antiserum was rendered specific by cross-absorbing it with the very mutant whose lack of antigen was being tested, we must ask whether such reasoning is not circular.

The use of this antiserum, developed for other reasons, is legitimized as follows. (i) The crude serum displays only one non-ODC band in immunoblots, a band seen in wild-type (strain IC-3) extracts and in those of the LV10-bearing strains. This band is absent from blots of a pure ODC preparation. The preimmune serum does not display reactivity to any polypeptide of the extract from strain IC-3. (ii) The exposure of crude antiserum to gel-immobilized extracts of the LV10-carrying strain was done at a very high ratio of immobilized antigen to antiserum. If ODC-related antigens (i.e., reactive nonsense fragments) were present in the immobilized antigen, the titer of ODC-reactive antibodies would have been diminished or lost entirely. No change of titer was seen. (iii) Immunotitrations done with mixtures of extracts of strain IC-3 and the LV10-bearing mutant show no 


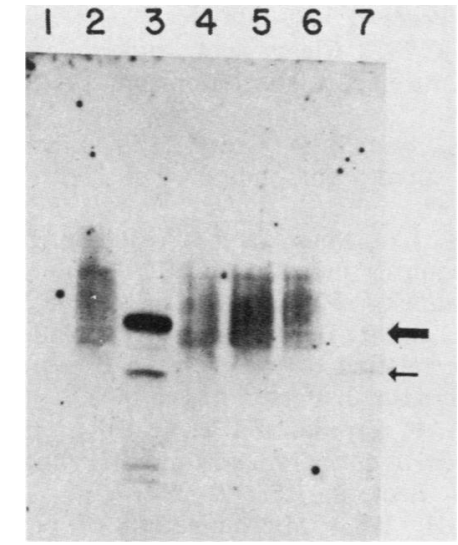

FIG. 3. Immunoblot of extracts, separated by sodium dodecyl sulfate-polyacrylamide gel electrophoresis and probed with the cross-absorbed anti-ODC antiserum. The extracts in each lane are from strains of the following genotypes: 1, LV10; 2, LV10 ssu-1; 3 , aga (strain IC3 starved for polyamines [control]); 4, PE4 ssu-1; 5, PE4 $s u^{123} ; 6$, PE4 $s u^{127} ; 7$, PE4. All suppressed mutants were grown in minimal medium; the PE4 and LV10 Spe ${ }^{-}$mutants were grown on limiting putrescine $(0.2 \mathrm{mM})$. The large arrow designates the position of pure ODC $\left(M_{\mathrm{r}}=53,000\right)$, which is visualized in the control lane. The small arrow gives the position of the mutant product in the PE4-bearing strain.

alteration of the slope of enzyme units removed per microliter of the crude antiserum, whereas similar mixtures of the arginase-less extract and those of an extract of strain IC-1897-24, bearing mutation LV86, show the expected competition (J. J. DiGangi et al., submitted). This finding

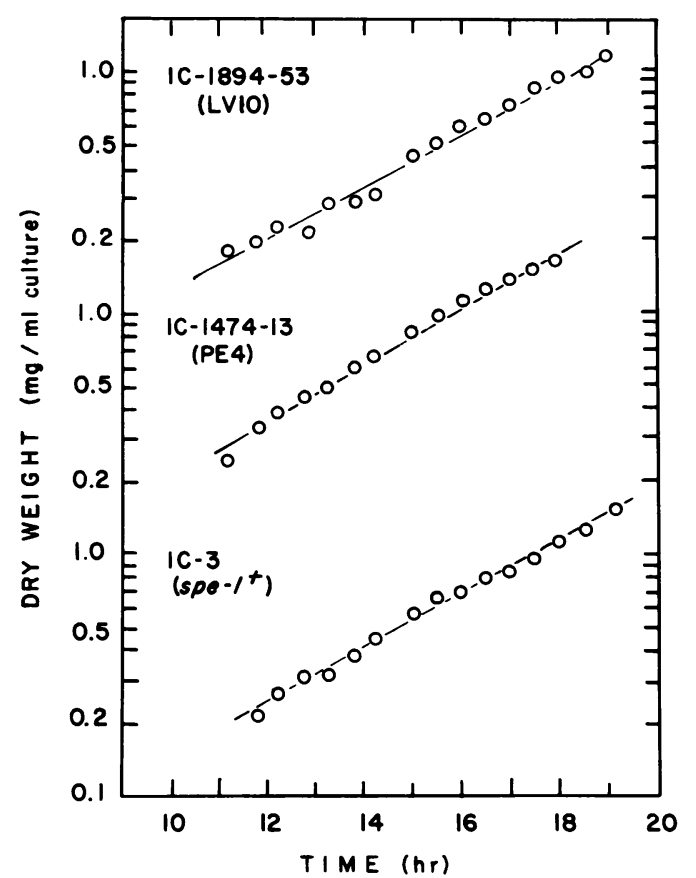

FIG. 4. Growth of strains IC-3, IC-1474-13, and IC-1894-53 bearing the wild-type spe-1 allele or the nonsense alleles PE4 and LV10, respectively, in spermidine-supplemented medium. The data are plotted on overlapping semilogarithmic scales. Doubling times for the cultures were $2.7,2.5$, and 2.8 for the spe-1+-, PE4-, and LV10-bearing strains, respectively. was true of three other crude antisera. (iv) No common antigen of the normal molecular weight or of less molecular weight in the extract of the LV10-carrying strain was displayed by the three other crude antisera, one to denatured ODC and two to native ODC. Immobilized extracts of the LV10-bearing strain did not reduce the titer of any of these three antisera. We feel that these reasons, together with the nonsense character of the LV10 mutation, legitimize the use of LV10 extracts in making ODC-specific antisera, as well as our conclusions regarding the absence of ODC protein in the strain carrying the LV10 mutation. This conclusion is compromised only by the possible persistence of an N-terminal fragment of ODC that is unrecognizable by antiserum but is indispensable for normal growth.

Because a mutant carrying a nonsense mutation and wholly lacking cross-reacting material is viable and healthy if it is given polyamine supplementation, we conclude that the asexual life cycle is not compromised by the lack of the ODC protein itself. The viability of two other strains without ODC protein (IC-1896-22 [carrying allele LV71] and IC-1901-14 [carrying allele LV170]), support this conclusion. The poor germination of the sexual spores (ascospores) might indicate that ODC has a role in the sexual cycle. However, ascospore germination is poor in all mutants with impaired ODC activity $(10,18)$ because polyamines provided in growth media penetrate poorly into cells of $N$. crassa (7). Thus it is likely that sterility is brought about by a polyamine deficiency, not by impairment of another ODC-related function. We therefore feel that the ODC protein is required only in the synthesis of polyamines. This conclusion may extend to mammalian cells in culture, because Steglich et al. (21) have reported variants of $\mathrm{CHO}$ cells lacking detectable ODC protein while retaining the ability to make ODC mRNA.

Polyamines have been shown to influence the fidelity of protein synthesis and to be required in some cases for the action of nonsense suppressor mutations in bacteria $(1,11$, $12,24,25)$. Our data show that nonsense suppression is qualitatively successful in $N$. crassa despite severe polyamine starvation in our suppressed mutants. However, the product of the suppressed-mutant or wild-type gene behaves peculiarly on immunoblots, even without our imposition of a polyamine limitation during growth of the suppressorbearing strains. We cannot interpret this finding presently, and it may have little to do with the process of translation.

Our data extend the evidence that spe-l is the structural gene for ODC. First, nonsense mutations allelic to those having defective ODC protein (10) are expected in an enzyme structural gene. Second, reversion of ODC-less mutants is rarely accompanied by restoration of normal ODC activity, as though second-site reversion within the gene happens frequently. The genetic data are consistent with intragenic events in such cases. The residual activity of extracts of strains bearing allele PE4 (10) shows that the active site of ODC is not encoded in the carboxy-terminal (ca. 85) amino acids of the protein, assuming that the mutant product seen in immunoblots is responsible for the ODC activity measured in crude extracts.

\section{ACKNOWLEDGMENTS}

We thank Janet Ristow for excellent technical assistance with immunoblotting and Daniel Knauer for the gift of ${ }^{125} \mathrm{I}$-labeled protein A.

This work was supported by research grant BC-366A from the American Cancer Society and Public Health Service research grant GM-35120 from the National Institute of General Medical Sciences. 


\section{LITERATURE CITED}

1. Algranati, I. D., and S. H. Goldemberg. 1981. Initiation, elongation and termination of polypeptide synthesis in cell-free systems from polyamine-deficient bacteria. Biochem. Biophys. Res. Commun. 103:8-15.

2. Bradford, M. M. 1976. A rapid and sensitive method for the quantitation of microgram quantities of protein utilizing the principle of dye binding. Anal. Biochem. 72:248-254.

3. Burnette, W. N. 1981. "Western blotting": electrophoretic transfer of proteins from sodium dodecyl sulfate-polyacrylamide gels to unmodified nitrocellulose and radiographic detection with antibody and radioiodinated protein A. Anal. Biochem. 112:195-203.

4. Case, M. E., and N. H. Giles. 1974. Revertants and secondary arom-2 mutants induced in non-complementing mutants in the arom gene cluster of Neurospora crassa. Genetics 77:613-621.

5. Cohn, M. S., C. W. Tabor, and H. Tabor. 1980. Regulatory mutations affecting ornithine decarboyxlase activity in Saccharomyces cerevisiae. J. Bacteriol. 142:791-799.

6. Davis, R. H., and F. J. de Serres. 1970. Genetic and microbiological research techniques for Neurospora crassa. Methods Enzymol. 17A:79-143.

7. Davis, R. H., G. N. Krasner, J. J. DiGangi, and J. L. Ristow. 1985. Distinct roles of putrescine and spermidine in the regulation of ornithine decarboxylase in Neurospora crassa. Proc. Natl. Acad. Sci. USA 82:4105-4109.

8. Davis, R. H., J. L. Ristow, and C. L. Ginsburgh. 1981. Independent localization and regulation of carbamyl phosphate synthetase A polypeptides of Neurospora crassa. Mol. Gen. Genet. 181:215-221.

9. Davis, R. H., and R. L. Weiss. 1983. Identification of nonsense mutations in Neurospora: application to the complex arg-6 locus. Mol. Gen: Genet. 192:46-50.

10. Eversole, P., J. J. DiGangi, T. Menees, and R. H. Davis. 1985. Structural gene for ornithine decarboxylase in Neurospora crassa. Mol. Cell. Biol. 5:1301-1306.

11. Igarishi, K., K. Kashiwagi, K. Kishida, T. Kakegawa, and S. Hirose. 1981. Decrease in the S1 protein of 30-S ribosomal subunits in polyamine-requiring mutants of Escherichia coli grown in the absence of polyamines. Eur. J. Biochem. 114:127-131.

12. Jorstad, C. M., and D. R. Morris. 1974. Polyamine limitation of growth slows the rate of polypeptide chain elongation in Escherichia coli. J. Bacteriol. 119:857-860.

13. Kuehn, G. D., and V. J. Atmar. 1982. Posttranslational control of ornithine decarboxylase by polyamine-dependent protein kinase. Fed. Proc. 41:3078-3083.

14. Laemmli, U. K. 1970 . Cleavage of structural proteins during the assembly of the head of bacteriophage T4. Nature (London) 27:680-685.

15. Lester, H. E., and S. R. Gross. 1959. Efficient method for selection of auxotrophic mutants of Neurospora. Science 129:572.

16. McDougall, K. J., J. Deters, and J. Miskimin. 1977. Isolation of putrescine-requiring mutants of Neurospora crassa. Antonie van Leeuwenhoek J. Microbiol. Serol. 43:143-151.

17. Paulus, T. J., and R. H. Davis. 1981. Regulation of polyamine synthyesis in relation to putrescine and spermidine pools in Neurospora crassa. J. Bacteriol. 145:14-20.

18. Paulus, T. J., P. Kiyono, and R. H. Davis. 1982. Polyaminedeficient Neurospora crassa mutants and synthesis of cadaverine. J. Bacteriol. 152:291-297.

19. Russell, D. H. 1983. Microinjection of purified ornithine decarboxylase into Xenopus oocytes selectively stimulates ribosomal RNA synthesis. Proc. Natl. Acad. Sci. USA 80:13181321.

20. Sneath, P. H. A. 1955. Putrescine as an essential growth factor for a mutant of Aspergillus nidulans. Nature (London) 175: 818-819.

21. Steglich, C., A. Grens, and I. E. Scheffler. 1984. Chinese hamster cells deficient in ornithine decarboxylase: reversion by gene amplification and by azacytidine treatment. Somatic Cell Genet. 11:11-23

22. Steglich, C., and I. E. Scheffier. 1982. An ornithine decarboxylase-deficient mutant of Chinese hamster ovary cells. J. Biol. Chem. 257:4603-4609.

23. Tabor, C. W., and H. Tabor. 1984. Polyamines. Annu. Rev. Biochem. 53:749-790.

24. Tabor, H., and C. W. Tabor. 1982. Polyamine requirement for efficient translation of amber codons in vivo. Proc. Natl. Acad. Sci. USA 79:7087-7091.

25. Tabor, H., C. W. Tabor, M. S. Cohn, and E. W. Hafner. 1981. Streptomycin resistance ( $r p s L)$ produces an absolute requirement for polyamines for growth of an Escherichia coli strain unable to synthesize putrescine and spermidine $[\Delta(s p e A-$ speB) $\Delta$ speC]. J. Bacteriol. 147:702-704.

26. Vogel, H. J. 1964. Distribution of lysine pathways among fungi: evolutionary implications. Am. Nat. 98:435-446.

27. Whitney, P. A., and D. R. Morris. 1978. Polyamine auxotrophs of Saccharomyces cerevisiae. J. Bacteriol. 134:214-220.

28. Young, R. A., and R. W. Davis. 1983. Yeast RNA polymerase II gene: isolation with antibody probes. Science 222:778-782. 